\title{
Madeiras tropicais quanto à densidade e cor para uso em pavimentação
}

\section{Tropical wood for density and color to use in paving}

Lílian Thais Cantanhêde Rocha; Mestranda em Design, UFMA. lilian.cantanhede@hotmail.com

Sanatiel de Jesus Pereira; Doutor, UFMA.

pereirasj@terra.com.br

Karoline de Lourdes Monteiro Guimarães; Mestranda em Design, UFMA.

kgguimaraes@hotmail.com

Mariana Sousa Valporto; Mestranda em Design, UFMA.

marianavalporto@gmail.com

Valkiria Aires Viegas; Mestranda em Design, UFMA.

valviegas@hotmail.com

Resumo: Objetivando descobrir as possíveis combinações de espécies de madeiras diferentes com base na relação densidade $x$ cor para a aplicação em pisos, desenvolvemos o presente estudo, no qual foram consideradas características como, dureza janka e retratibilidade das 95 espécies pesquisadas até chegarmos ao grupo de 30 espécies. Destas, com base na densidade e na cor de cada uma, constatamos que as madeiras mais escuras são também as mais pesadas. Verificamos ainda que, nem todas as espécies mais escuras podem ser combinadas com as mais claras, visto que possuem uma grande variação de densidade, o que irá interferir na retratibilidade das espécies.

Palavras-chave: Madeiras; propriedades; pisos.

Abstract: Aiming to discover the possible combinations of species of different woods from the relation color $x$ density for use in floors, developed the present study, which were considered as features, Janka hardness and shrinkage of 95 species surveyed until we reach the group of 30 species. Of these, based on the density and color of each, found that the darker wood are also heavier. We have also noticed that not all the darker species may be combined with the lighter since they are a large variation in density, which will interfere with the shrinkage of species.

Keywords: Woods; properties; floors. 


\section{INTRODUÇÃO}

Desde os primórdios da humanidade, a madeira tem sido um dos mais versáteis e difundidos materiais utilizados pelo homem. Nos dias atuais é usada na produção de móveis, na construção civil (estacas, formas e andaimes), como componentes de acabamento em unidades habitacionais (portas, janelas, assoalhos, guarnições, rodapés, dentre outras aplicações), em estruturas como pontes e passarelas, utilidades como escadas portáteis, cabos de ferramentas, objetos de arte e de decoração, e uma infinidade de outros usos.

Por se tratar de uma matéria-prima importante e versátil em vários setores da atividade humana, a madeira pode ser aplicada em diversas situações, sendo destaque entre outros materiais por apresentar diversas propriedades como, a necessidade de um baixo consumo de energia para o seu processamento, a alta resistência específica, as boas características de isolamento térmico e elétrico, além de ser um material muito fácil de ser trabalhado manualmente ou por máquinas.

A madeira é um dos produtos mais valiosos que as árvores oferecem através da retirada de seu tronco arbóreo, que se compõe em duas porções fundamentais: 0 alburno (viva e externa) e o cerne (morta e interna). Sob o aspecto comercial, entretanto, a madeira propriamente dita é somente o cerne, em virtude de suas diversas qualidades.

Sendo durável e resistente, tal material torna-se sinônimo de conforto e aconchego quando aplicado em pisos, podendo ser empregado a partir da combinação de sua cor, desenho, grã, brilho e outros. Características que variam tanto de espécie para espécie quanto de árvore para árvore, como destaca Galina (et al., 2013, p. 02):

“[...] a madeira apresenta variações na estrutura anatômica, na composição química e nas propriedades físicas e mecânicas, não apenas entre as diversas espécies arbóreas (diferenças acentuadas e marcantes) como também entre indivíduos de uma mesma espécie e até entre peças obtidas de um mesmo indivíduo."

A Figura 1 mostra um exemplo de combinação aleatória de espécies de madeira a partir de suas cores e desenhos e a Figura 2 ilustra uma combinação de piso a partir das cores das madeiras.

Figura 1 - Madeiras combinadas aleatoriamente

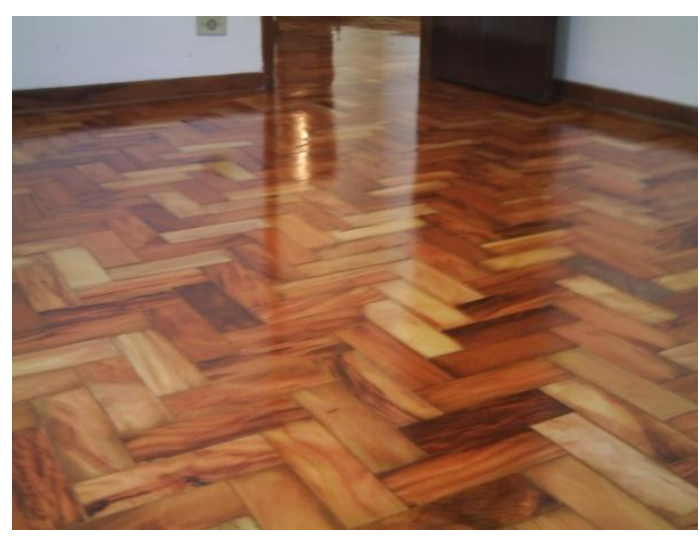

Fonte: adaptado de www.portaldasideias.org
Figura 2 - Madeiras combinadas por cor

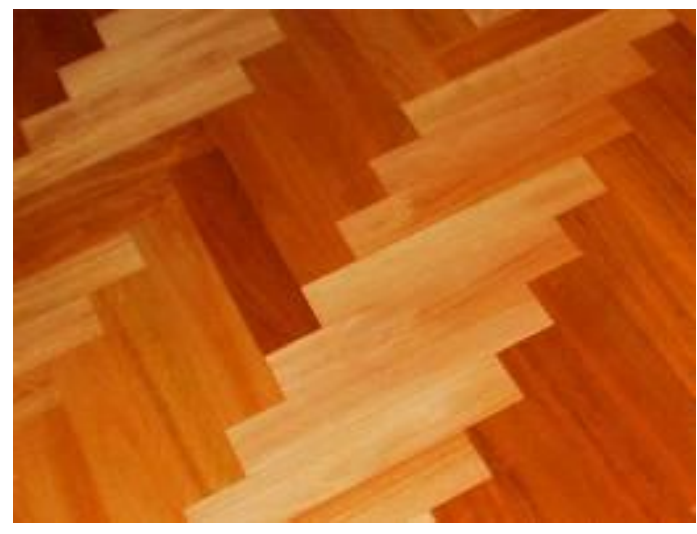

Fonte: adaptado de www.portaldasideias.org

Mori (et al., 2004) aponta que características como a densidade, textura, grã e dureza são bastante utilizadas na classificação da madeira e que, recentemente, a cor também vem sendo estabelecida como índice para tal. Nesta via, Estuqui Filho (2006) aponta 
que as madeiras escuras são as mais duráveis e dispensam tratamento, enquanto as claras necessitam de maiores cuidados com relação à proliferação de fungos que causam manchas e o apodrecimento do material.

Vários autores apontam que a variação de cor entre as diversas espécies de madeira depende tanto da quantidade de extrativos presentes na parede celular do cerne quanto da influência de fatores externos como a alteração do teor de umidade durante um processo de secagem, por exemplo. No presente trabalho, trataremos da cor a partir de sua concepção estética a fim de que se obtenham resultados de combinação de espécies a partir de sua relação com a densidade.

Segundo Galina (et. al. 2013), considerando características como dimensões, espécie, acabamento superficial e presença/ausência de encaixes nas laterais, os pisos podem ser classificados em 02 grupos principais: maciços e engenheirados.

Dentre os pisos maciços, temos:

- Assoalho: peças de madeira maciça variando entre 08 a $22 \mathrm{~mm}$ de espessura, largura entre 57 e $210 \mathrm{~mm}$ e comprimentos variando entre 280 e $6000 \mathrm{~mm}$. Tais peças apresentam encaixes macho/fêmea em 02 ou 04 laterais.

- Taco: peças de madeira maciça com ou sem encaixes macho/fêmea nas laterais, espessura entre 08 e $20 \mathrm{~mm}$, largura e comprimento fixos podendo ser confeccionados em várias dimensões. Geralmente as dimensões do comprimento são múltiplas em relação à largura.

- Parquet: são várias peças maciças unidas, formando placas quadradas de 240 x 240 $\mathrm{mm}, 482 \times 482 \mathrm{~mm}$ ou de dimensões e formatos variados. Sua espessura pode variar entre $06 \mathrm{~mm}$ e $18 \mathrm{~mm}$. Também podem ser chamados de parquet mosaico, devido à possibilidade de formação de diferentes desenhos no momento da instalação.

Quanto aos pisos engenheirados:

- Piso Estruturado: geralmente esses tipos de pisos já são envernizados na fábrica e podem ser classificados em três tipos, ou seja, estruturado maciço, estruturada lamela e estruturado lâmina.

- Piso laminado: são painéis de fibras ou partículas de madeira reconstituída, como MDF (painel de fibras de média densidade), HDF (painel de fibras de alta densidade) e MDP (painel de partículas de média densidade). Os painéis são revestidos por uma camada de papel impregnado com resina melamínica, que proporciona variados padrões decorativos inclusive imitando a madeira natural.

A escolha da matéria-prima no desenvolvimento do projeto de um produto é uma das etapas mais importantes para que o produto tenha seus requisitos de funcionamento preenchidos. Entretanto, a falta de conhecimento tecnológico adequado pode resultar em produtos estruturalmente deficientes comprometendo assim as reais potencialidades da matéria-prima. Nesta via, a fim de que se obtenham produtos com desempenhos mais satisfatórios, tanto no aspecto estrutural quanto econômico, bem como da vida útil dos mesmos, insere-se tanto o designer quanto o arquiteto como mão-de-obra especializada na confecção de projetos.

Em se tratando de madeira, dentre o leque de valores positivos que esta proporciona, Galina (et. al. 2013) aponta que, para a seleção de uma espécie como adequada à fabricação de pisos, deve-se considerar, principalmente, as propriedades físicomecânicas como massa específica, dureza janka e retratibilidade. Adicionalmente, podem-se considerar ainda os aspectos estéticos e as tendências da moda. 
Conforme a definição da ABNT (apud Galina, 2013), a massa específica da madeira é a relação entre a massa e o volume do material quando o teor de umidade é de $15 \%$. Usualmente a massa específica é expressa em gramas por centímetro cúbico $\left(\mathrm{g} / \mathrm{cm}^{3}\right)$ ou quilogramas por metro cúbico $\left(\mathrm{kg} / \mathrm{m}^{3}\right)$.

Sendo assim, como aponta Rodrigues (1996) a massa específica aparente, ou ainda densidade, é a expressão mais exata da concentração e da distribuição de material resistente na estrutura da madeira. Neste contexto, pode-se dizer que todas as características físicas e mecânicas das madeiras estão diretamente relacionadas com sua massa específica aparente.

A dureza janka, como define Rodrigues (1996), é a resistência do material à penetração localizada, à riscagem e ao desgaste.

A outra característica aqui destacada é a retratibilidade. Na concepção de Galina (et. al., 2013), trata-se do conjunto das características de retração e inchamento da madeira. A variação dimensional em qualquer uma das três direções estruturais da madeira (longitudinal, radial e tangencial) é calculada como percentagem de variação em relação à dimensão inicial.

A retração e o inchamento da madeira estão diretamente relacionados com o seu teor de umidade. $O$ inchamento ou aumento no volume é resultante de um aumento no teor de umidade da madeira. No sentido inverso, a retração ou redução no volume verifica-se quando ocorre redução no teor de umidade da madeira.

Com o intuito de propor combinações de espécies a partir da densidade e cor, a fim de que estas sejam aplicadas em pisos, selecionamos 30 espécies considerando sua dureza janka e coeficiente de retratibilidade satisfatórios.

Concomitantemente, a partir das espécies selecionadas destacamos suas densidades e classificamos cada espécie por cor conforme categorias sugeridas pelos autores deste estudo.

\section{DESENVOLVIMENTO}

\subsection{Material e métodos}

Os materiais utilizados para a realização deste estudo foram extraídos do livro Fichas de Características das madeiras brasileiras. Neste livro o autor recomenda 95 espécies de madeira que julgou ideais para a utilização em pisos. Destas, constatamos que apenas 88 espécies apresentam fichas completas, o que indica que foram desenvolvidos todos os estudos necessários para a coleta de dados.

Neste grupo de 88 espécies verificamos que 30 delas estão distribuídas aos pares apresentando os mesmos dados, nas mesmas fichas, variando apenas suas nomenclaturas. Deste modo, consideramos apenas a primeira nomenclatura atribuída em cada ficha. Assim, reduzimos o grupo para 73 espécies. Feito isto, analisamos cada espécie a partir de sua dureza janka e destacamos apenas aquelas que possuem dureza janka alta, conforme classificação destacada por Rodrigues (1996) no Quadro 1. Das 73 espécies analisadas, resultaram 41. 
Quadro 1 - Classificação - Dureza janka

\begin{tabular}{|c|c|}
\hline Classificação & Dureza Janka - madeira verde (kgf) \\
\hline Alto & Acima de 700 \\
\hline Médio & 400 a 700 \\
\hline Baixo & Abaixo de 400 \\
\hline
\end{tabular}

Fonte: elaborado pelo autor com base em Rodrigues (1996).

Como destaca Galina (et al., 2013), um dos índices utilizados na seleção de espécies para a aplicação em pisos é denominado relação $T / R$, que é a relação entre os valores máximos de retração nos sentidos tangencial $(T)$ e radial $(R)$.

Nesse sentido, espécies ou tipos de madeiras cujo índice T/R é inferior a 1,5 significa que são madeiras mais homogêneas quanto à variação dimensional, e que tenderão a apresentar deformações pequenas em resposta à variação sazonal no teor de umidade da madeira.

Por outro lado, espécies com índice T/R superiores a 2,5 são madeiras que tenderão a apresentar deformações comparativamente maiores em função das mudanças climáticas ao longo do ano.

Como critério de eliminação e, considerando que a maior variação de deformações poderá dificultar a combinação de espécies por cor, descartamos todas as que apresentaram a relação $T / R$ superior a 2,0. Deste modo, obtivemos 30 espécies para o estudo. O Quadro 2 traz os valores de relação $T / R$, dureza janka e massa específica aparente de cada espécie.

Quadro 2 - Espécies estudadas quanto à Relação T/R, Dureza Janka e Me

\begin{tabular}{|c|c|c|c|c|c|c|}
\hline \multirow{2}{*}{ Nome Popular } & \multirow{2}{*}{ Nome Científico } & \multicolumn{2}{|c|}{ Retrações (\%) } & \multirow{2}{*}{$\begin{array}{c}\text { Relação } \\
\text { T/R }\end{array}$} & \multirow{2}{*}{$\begin{array}{c}\text { Dureza } \\
\text { Janka } \\
\text { (kgf) }\end{array}$} & \multirow{2}{*}{$\begin{array}{c}\text { Me Ap. } \\
(15 \% \text { - } \\
\left.\mathrm{g} / \mathrm{cm}^{3}\right)\end{array}$} \\
\hline & & Tangencial & Radial & & & \\
\hline Achuarana & Vantanea cupularis Hub. & 10,50 & 10,40 & 1,01 & 989 & 1,07 \\
\hline Sucupira-açu & Diplotropis incexis Rizz. \& Matt & 5,60 & 4,40 & 1,27 & 787 & 0,80 \\
\hline Acapu & Vouacapoua americana Aubl. & 7,10 & 4,90 & 1,45 & 741 & 0,91 \\
\hline Ipê-pardo & Tabebuia ochracea (Cham.) Rizz. & 5,90 & 4,00 & 1,48 & 1.102 & 1,01 \\
\hline Baru & Dipteryx alata Vog. & 7,30 & 4,90 & 1,49 & 1.191 & 1,10 \\
\hline Sucupira-parda & Bowdichia virgilioides H.B.K. & 8,40 & 5,40 & 1,56 & 847 & 0,91 \\
\hline Carne-de-vaca & Vantanea $s p$ & 12,50 & 8,00 & 1,56 & 848 & 0,96 \\
\hline Gonçalo-alves & Astronium macrocalyx Engl. & 9,30 & 5,90 & 1,58 & 933 & 1,07 \\
\hline Maçaranduba & Manilkara longifolia (A.DC.) Dub. & 11,00 & 6,80 & 1,62 & 980 & 1,00 \\
\hline Óleo-pardo & Myrocarpus sp & 11,10 & 6,80 & 1,63 & 1.023 & 1,01 \\
\hline Canela-preta & Acrodicridium $s p$ & 9,80 & 6,00 & 1,63 & 1.019 & 0,99 \\
\hline Pequiá & Aspidosperma sp & 8,90 & 5,40 & 1,65 & 927 & 0,83 \\
\hline Angico-preto & $\begin{array}{c}\text { Anadenanthera macrocarpa (Benth.) } \\
\text { Brenae }\end{array}$ & 8,10 & 4,90 & 1,65 & 1.175 & 1,05 \\
\hline Ipê-roxo & $\begin{array}{l}\text { Tabebuia impetiginosa (Mart.) } \\
\text { Standl. }\end{array}$ & 7,20 & 4,30 & 1,67 & 885 & 0,96 \\
\hline Bálsamo & Myroxylon balsamum (L.) Harms. & 6,70 & 4,00 & 1,68 & 1.034 & 0,95 \\
\hline Amarelinho & Helietta longifoliata Britt. & 10,40 & 6,20 & 1,68 & 828 & 0,98 \\
\hline Sucupira-amarela & Ferreirea spectabilis Fr. Aliem. & 7,00 & 4,10 & 1,71 & 922 & 0,99 \\
\hline
\end{tabular}




\begin{tabular}{|l|c|c|c|c|c|c|} 
Pau-cepilho & \multicolumn{1}{|c|}{ Vantanea sp } & 11,80 & 6,90 & 1,71 & 789 & 0,91 \\
\hline Guatambu-peroba & Aspidosperma populifolium A.DC. & 9,60 & 5,50 & 1,75 & 770 & 0,82 \\
\hline Guaribu-amarelo & Goniorrhachis marginata Tul. & 8,40 & 4,80 & 1,75 & 964 & 1,01 \\
\hline Pau-roxo & Peltogyne recifensis Ducke & 7,90 & 4,40 & 1,80 & 1.401 & 1,13 \\
\hline Cuvantã & Cupania vernalis Camb. & 10,50 & 5,80 & 1,81 & 732 & 0,82 \\
\hline Jataí-peba & Dialium guianense (Aubl.) Sandw. & 11,50 & 6,30 & 1,83 & 1.420 & 1,12 \\
\hline Jacarandá-do-litoral & Platymiscium floribundum Vog. & 7,20 & 3,90 & 1,85 & 789 & 0,89 \\
\hline Gibatão & Astronium graveolens Jacq. & 8,00 & 4,30 & 1,86 & 834 & 0,97 \\
\hline Amoreira & Chlorophora tinctoria (L.) Gaud. & 4,30 & 2,30 & 1,87 & 1.038 & 0,88 \\
\hline Aroeira-do-sertão & Astronium urundeuva (Fr. Aliem.) & 7,20 & 3,80 & 1,89 & 1.126 & 1,19 \\
\hline Garapa & Apuleia leiocarpa (Vog.) Macbr. & 8,50 & 4,40 & 1,93 & 740 & 0,83 \\
\hline Faveiro & Pterodon pubescens Benth. & 6,80 & 3,50 & 1,94 & 963 & 0,94 \\
\hline Coração-de-negro & Poecilanthe parviflora Benth. & 8,00 & 4,00 & 2,00 & 1.223 & 0,99 \\
\hline
\end{tabular}

Fonte: elaborado pelo autor com base na pesquisa realizada.

Galina (et. al., 2013, p. 04) destaca que "madeiras com massa específica inferior a 500 $\mathrm{kg} / \mathrm{m}^{3}$ são consideradas leves, e as com massa específica acima de $750 \mathrm{~kg} / \mathrm{m}^{3}$ são consideradas pesadas. As madeiras com massa específica na faixa intermediária (de 500 a $750 \mathrm{~kg} / \mathrm{m}^{3}$ ) são tidas como médias".

A partir da classificação de Rodrigues (1996) temos que madeiras com densidade inferior a 0,5 são consideradas leves enquanto aquelas que apresentam valores superiores a 1,0 são classificadas como muito pesadas, conforme o Quadro 3.

Quadro 3 - Classificação - Densidade

\begin{tabular}{|c|c|}
\hline Classificação & Densidade \\
\hline Leves & Menor que 0,05 \\
\hline Moderadamente pesadas & 0,05 a 0,75 \\
\hline Pesadas & 0,75 a 0,99 \\
\hline Muito pesadas & Acima de 1,00 \\
\hline
\end{tabular}

Fonte: elaborado pelo autor com base em Rodrigues (1996).

Noutra via, em se tratando de cor, categorizamos as espécies em: claras, escuras e muito escuras. Assim, as madeiras que apresentam o cerne com coloração bege, amarelada ou castanho-claro foram classificadas como claras; as que apresentam variação de cor entre as colorações acima citadas e os tons castanhos ou róseoacastanhados foram classificadas em escuras; as de coloração mais intensa, roxa, tendendo para o castanho-escuro ou castanho-avermelhado são classificadas em escuras.

Deste modo, a partir do Quadro 4, temos:

Quadro 4 - Classificação de espécies quanto à cor e Me Ap. (densidade)

\begin{tabular}{|l|c|c|c|c|}
\hline \multicolumn{1}{|c|}{ Nome Popular } & Nome Científico & Cor & $\begin{array}{c}\text { Me Ap. (15\% } \\
\left.-\mathbf{g} / \mathbf{c m}^{\mathbf{3}}\right)\end{array}$ & Classificação \\
\hline Baru & Dipteryx alata Vog. & Clara & 1,10 & Muito pesada \\
\hline Achuarana & Vantanea cupularis Hub. & Clara & 1,07 & Muito pesada \\
\hline
\end{tabular}




\begin{tabular}{|c|c|c|c|c|}
\hline Ipê-pardo & Tabebuia ochracea (Cham.) Rizz. & Clara & 1,01 & Muito pesada \\
\hline Coração-de-negro & Poecilanthe parviflora Benth. & Clara & 0,99 & Pesada \\
\hline Amarelinho & Helietta longifoliata Britt. & Clara & 0,98 & Pesada \\
\hline Pau-cepilho & Vantanea sp & Clara & 0,91 & Pesada \\
\hline Amoreira & Chlorophora tinctoria (L.) Gaud. & Clara & 0,88 & Pesada \\
\hline Pequiá & Aspidosperma sp & Clara & 0,83 & Pesada \\
\hline Cuvantã & Cupania vernalis Camb. & Clara & 0,82 & Pesada \\
\hline Guatambu-peroba & Aspidosperma populifolium A.DC. & Clara & 0,82 & Pesada \\
\hline Gonçalo-alves & Astronium macrocalyx Engl. & Escura & 1,07 & Muito pesada \\
\hline Angico-preto & $\begin{array}{l}\text { Anadenanthera macrocarpa (Benth.) } \\
\text { Brenae }\end{array}$ & Escura & 1,05 & Muito pesada \\
\hline Óleo-pardo & Myrocarpus sp & Escura & 1,01 & Muito pesada \\
\hline Canela-preta & Acrodicridium $s p$ & Escura & 0,99 & Pesada \\
\hline Sucupira-amarela & Ferreirea spectabilis Fr. Aliem. & Escura & 0,99 & Pesada \\
\hline Gibatão & Astronium graveolens Jacq. & Escura & 0,97 & Pesada \\
\hline Carne-de-vaca & Vantanea sp & Escura & 0,96 & Pesada \\
\hline Ipê-roxo & Tabebuia impetiginosa (Mart.) Standl. & Escura & 0,96 & Pesada \\
\hline Bálsamo & Myroxylon balsamum (L.) Harms. & Escura & 0,95 & Pesada \\
\hline Faveiro & Pterodon pubescens Benth. & Escura & 0,94 & Pesada \\
\hline $\begin{array}{l}\text { Jacarandá-do- } \\
\text { litoral }\end{array}$ & Platymiscium floribundum Vog. & Escura & 0,89 & Pesada \\
\hline Garapa & Apuleia leiocarpa (Vog.) Macbr. & Escura & 0,83 & Pesada \\
\hline Sucupira-açu & Diplotropis incexis Rizz. \& Matt & Escura & 0,80 & Pesada \\
\hline Aroeira-do-sertão & Astronium urundeuva (Fr. Aliem.) Engl. & Muito Escura & 1,19 & Muito pesada \\
\hline Pau-roxo & Peltogyne recifensis Ducke & Muito Escura & 1,13 & Muito pesada \\
\hline Jataí-peba & Dialium guianense (Aubl.) Sandw. & Muito Escura & 1,12 & Muito pesada \\
\hline Guaribu-amarelo & Goniorrhachis marginata Tul. & Muito Escura & 1,01 & Muito pesada \\
\hline Maçaranduba & Manilkara longifolia (A.DC.) Dub. & Muito Escura & 1,00 & Muito pesada \\
\hline Acapu & Vouacapoua americana Aubl. & Muito Escura & 0,91 & Pesada \\
\hline Sucupira-parda & Bowdichia virgilioides H.B.K. & Muito Escura & 0,91 & Pesada \\
\hline
\end{tabular}

Fonte: elaborado pelo autor com base na pesquisa realizada.

\section{RESULTADOS E DISCUSSÃO}

Conforme o Quadro 4, das 30 espécies selecionadas, temos que 11 possuem altos valores de densidade e, portanto, são consideradas "muito pesadas". Quanto à cor, 10 são claras, 13 são escuras e 7 são muito escuras. Constatamos ainda que a maioria das espécies muito escuras são também mais pesadas. Estas madeiras consideradas muito pesadas apresentam maior resistência a impactos que as demais.

Notamos ainda que, nem toda espécie que apresenta um alto valor de massa específica irá necessariamente apresentar um alto índice de relação T/R. Como exemplo, destacamos as espécies Aroeira-do-sertão e Coração-de-negro. A primeira apresenta uma massa específica de $1,19 \mathrm{~g} / \mathrm{cm}^{3}$ e seu índice $T / R$ é de 1,89 , enquanto a segunda apresenta valores equivalentes a $0,99 \mathrm{~g} / \mathrm{cm}^{3}$ e 2,00, respectivamente.

Noutra via, notamos que quanto menor é a variação de valores de massa específica de duas espécies, menor será a variação do índice $T / R$ destas. $O$ que permite destacar que 
a combinação mais viável de espécies poderá ser feita a partir da proximidade de seus valores de massa específica aparente. Se os valores de índice $T / R$ forem mais compatíveis, a variação de retratibilidade das espécies também será, o que permitirá que tais espécies proporcionem melhores encaixes.

A Figura 3 ilustra a relação Densidade x Cor das espécies selecionadas.

Figura 3 - Gráfico Densidade x Cor

\section{Densidade x Cor}

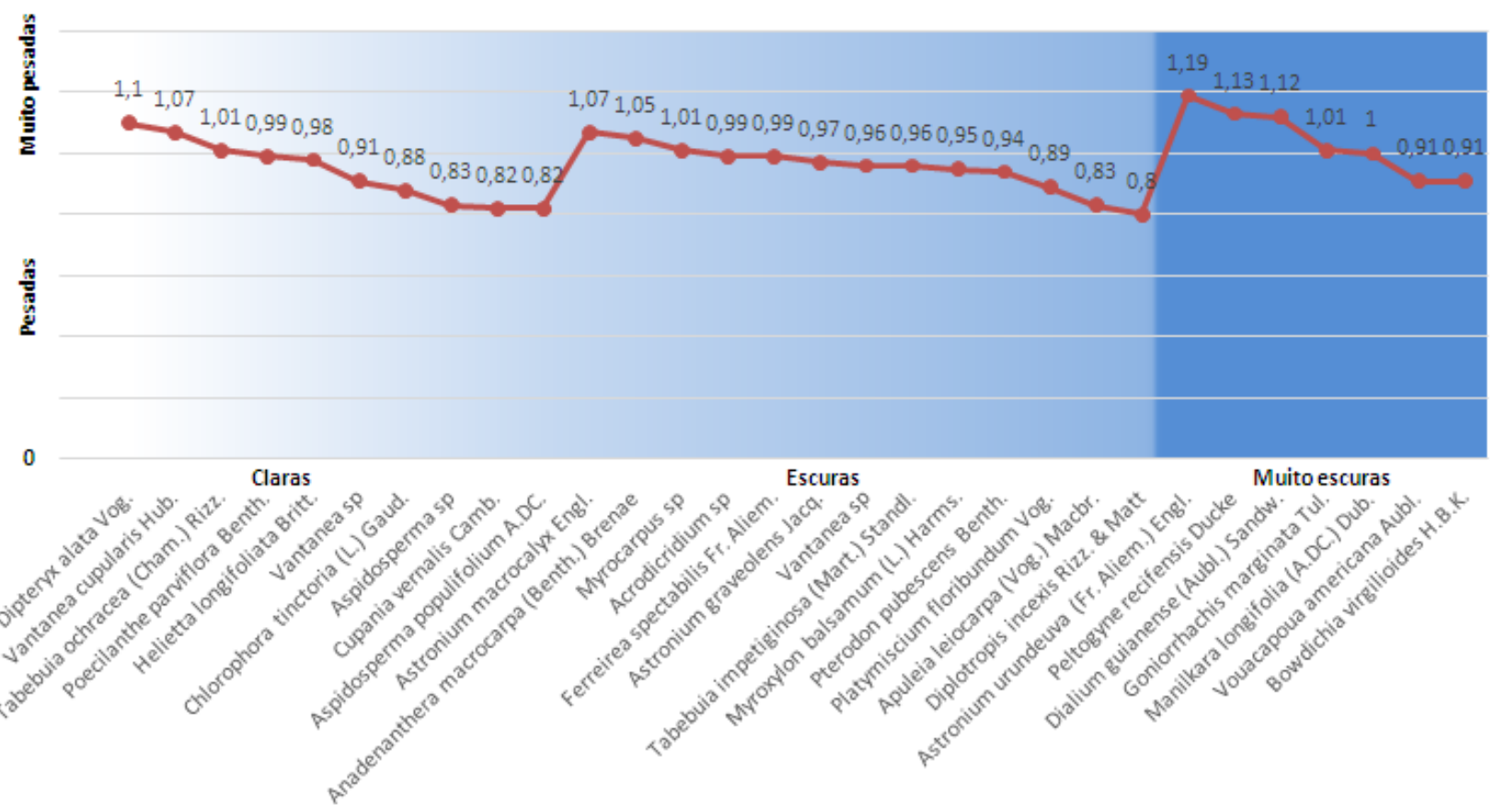

Fonte: elaborado pelo autor com base na pesquisa realizada.

A partir do gráfico temos que a combinação entre as espécies Vantanea sp. (clara com densidade de $0,91 \mathrm{~g} / \mathrm{cm}^{3}$ ) e Vouacapoua americana Aubl. (escura com densidade de $0,91 \mathrm{~g} / \mathrm{cm}^{3}$ ) poderá ser mais satisfatória que a combinação entre as espécies Cupania vernalis Camb. (clara com densidade de $0,82 \mathrm{~g} / \mathrm{cm}^{3}$ ) e Astronium urundeuva (Fr. Aliem.) Engl. (escura com densidade de $1,19 \mathrm{~g} / \mathrm{cm}^{3}$ ), pois apesar de apresentarem cores diferentes, a variação de densidade entre a primeira combinação será menor que a variação da segunda, permitindo assim um melhor encaixe entre as peças de espécies diferentes.

\section{CONCLUSÃO}

As madeiras estudadas apresentam Massa Específica Aparente (densidade) variando de 0,8 a $1,19 \mathrm{~g} / \mathrm{cm}^{3}$ sendo classificadas como pesadas e muito pesadas e, portanto, conforme autores revisados, ideais para a aplicação em pisos.

Um dos principais inconvenientes no uso da madeira em pisos é a sua movimentação dimensional, devido à troca de umidade entre a madeira e o ar. Quando a madeira ganha umidade ocorre um aumento nas suas dimensões (inchamento), verificando-se o fenômeno inverso (retração) quando a madeira perde umidade para o ar.

A capacidade que determinada madeira tem de variar suas dimensões é medida pelo índice T/R. Neste estudo, verificamos que os índices variaram de 1,01 a 2,00\%, o que 
indica que a maioria das espécies estudadas apresenta deformações pequenas em resposta à variação sazonal no teor de umidade da madeira.

Assim, se o propósito é combinar espécies a partir de suas cores de modo eficiente, deve-se considerar dentre outras propriedades, a massa específica aparente (densidade) que, no presente estudo, foi fator determinante na escolha de espécies a combinar.

Nesta via, constatamos que nem todas as espécies que apresentam colorações diferentes podem ser satisfatoriamente combinadas, visto que suas densidades são bem distintas, resultando em peças com diferentes variações de suas dimensões dificultando assim seus encaixes.

\section{REFERÊNCIAS}

ANDRADE, Ariel de at al. Auditoria em certificação da qualidade para pisos de madeira. Projeto Piso de Madeira Sustentável. São Paulo: Piracicaba, 2013. Disponível em: < http://www.pimads.org/>. Acesso em: 10 fev. 2014.

INSTITUTO DE PESQUISAS TECNOLÓGICAS DO ESTADO DE SÃO PAULO S. A. Madeira: uso sustentável na construção civil. São Paulo: SindusCon, 2003. Disponível em: < http://www.sindusconsp.com.br/downloads/prodserv/publicacoes/manual_madeira2. pdf>. Acesso em: 12 fev. 2014.

ESTUQUI FILHO, Carlos Adalberto. A durabilidade da madeira na arquitetura sob os fatores naturais: estudo de casos em Brasília. 2006. 148 f. Dissertação (Mestrado em Arquitetura e Urbanismo) - Programa de Pós-Graduação da FAU, Universidade de Brasília, Brasília, 2006.

GALINA, Inês Cristina Martins et al. Instalação de pisos de madeira. Projeto Piso de Madeira Sustentável. São Paulo: Piracicaba, 2013. Disponível em: < http://www.pimads.org/>. Acesso em: 10 fev. 2014.

GONZAGA, Armando Luiz. Madeira: uso e conservação. Brasília: IPHAN/Monumenta, 2006.

LISTA de espécies vegetais. São Paulo: Universidade Federal de São Carlos, [2013]. Disponível em: < http://www.ufscar.br/ debe/reserva/paginas/projeto/vegetais.pdf>. Acesso em: 15 jun. 2013.

MADEIRAS.

em: <http://www.aguademeninos.com.br/Madeireira/Madeiras/balsamo.html>. Acesso em: 17 jun. 2013.

MADEIRAS. Disponível em: <http://www.cobrire.com.br/deck-de-bambu.htm>. Acesso em: 17 jun. 2013.

MAINIERI, Calvino; CHIMELO, João Peres. Fichas de características das madeiras brasileiras. São Paulo: Instituto de Pesquisas Tecnológicas, 1989. 
MORESCHI, José Carlos. Propriedade da madeira. Departamento de Engenharia e Tecnologia Florestal da UFPR. Disponível em: < http://www.madeira.ufpr.br/publicacoes/propriedadesdamadeira2010.pdf>. Acesso em: 03 mar. 2014.

MORI, Cláudia L. S. de O. et al. Caracterização da cor da madeira de clones de híbridos de Eucalyptus spp. Cerne. Ci. Florestais, Lavras, v. 11, n. 2, p. 137-146, abr./jun. 2005. Disponível em: < http://www.dcf.ufla.br/cerne/artigos/v11_n2_artigo\%2004.pdf>. Acesso em: 13 fev. 2014.

MORI, Cláudia L. S. de O. et al. Influência das características tecnológicas na cor da madeira de Eucaliptos. Coral. Ciência Florestal, Santa Maria, v. 14, n. 2, p. 123-132, jun. $2004 . \quad$ Disponível em: < http://coral.ufsm.br/cienciaflorestal/artigos/v14n2/A12V14N2.pdf>. Acesso em: 13 fev. 2014.

PEREIRA, Sanatiel J. Pequeno dicionário de ciência e tecnologia da madeira. São Luís: Edufma, 2010.

PISOS de madeira. Disponível em: <http://www.remade.com.br/br/revistadamadeira_materia.php?num=976\&subject=Pi sos>. Acesso em: 15 jun. 2013.

RODRIGUES, Márcio José. Sistematização das propriedades da madeira para o uso racional no desenvolvimento de projeto de produto. $1996.125 \mathrm{p}$. Trabalho de Conclusão de Curso (Graduação em Desenho Industrial) para obtenção do grau de Bacharel em Desenho Industrial - Universidade Federal do Maranhão, São Luís, 1996.

SILVA, Moema Ribas. Materiais de Construção. São Paulo: Pini, 1985.

SILVA FILHO, Danilo Fernandes da et al. Influência da densidade na dureza janka em oito espécies madeireiras da Amazônia Central. Acta Amazônica, 22, 275-283, 1992. Disponível em: < https://acta.inpa.gov.br/fasciculos/22-2/PDF/v22n2a09.pdf>. Acesso em: 03 mar. 2014.

TIPOS de madeiras usadas em pisos de madeira. Disponível em: $<$ http://www.servicosempisosdemadeira.com.br/madeiras-para-pisos-demadeira.php>. Acesso em: 15 jun. 2013.

ZENID, Geraldo José. Madeira: uso sustentável na construção civil. São Paulo: Instituto de Pesquisas Tecnológicas - SVMA, 2009. 